\title{
Automatic skin lesion segmentation with optimal colour channel from dermoscopic images
}

\author{
Alaa Ahmed Abbas Al-abayechi ${ }^{\mathrm{a}, \mathrm{b}}$, Xiaoning Guo ${ }^{\mathrm{a}}$, Wooi-Haw Tan ${ }^{\mathrm{a}}$, Hamid A. Jalab ${ }^{\mathrm{c}, *}$ \\ ${ }^{a}$ Faculty of Engineering, Multimedia University, Cyberjaya, Malaysia \\ b Foundation of Technical Education, Institute of Administration Al-Rassafa Baghdad-Iraq \\ c Faculty of Computer Science and Information Technology, University of Malaya, Kuala Lumpur, 50603, \\ Malaysia
}

*Corresponding author, e-mail: hamidjalab@um.edu.my

\begin{abstract}
This article provides an improved automated skin lesion segmentation method for dermoscopic images. There are several stages for this method. These include the pre-processing steps such as resizing the images and eliminating noise. Hair was removed and reflective light was reduced using morphological operations and a median filter. The single green channel was rescaled into new intensities, as it provided the highest segmentation accuracy. The threshold value was calculated to separate the skin lesion region from healthy skin. Morphological operations were implemented to merge the small lesion areas around the bigger lesion areas with similar features and trace the boundary of the melanoma. The accuracy of the segmentation was evaluated by comparing the automatic boundary and manual boundary. Compared to other studies, our proposed method achieved the highest average accuracy of $97 \%$.
\end{abstract}

KEYWORDS: melanoma, skin cancer, image processing, dermatoscopy

\section{INTRODUCTION}

Melanoma is the most deadly form of skin cancer as it can develop quickly to spread to other organs and lymph nodes ${ }^{1}$. According to the American Cancer Society, the 10-year survival rates of this type of cancer can be as high as $86 \%$ if found during stage I. This figure was calculated based on nearly 60000 patients in the melanoma staging database. The survival rate declines exponentially after it has progressed to stage III. For this reason, early detection of melanoma plays a crucial role in increasing the survival rate for the patients ${ }^{2}$. Diagnosis of melanoma in skin lesions is an objective procedure performed by experienced dermatologists by sight. Computerised image analysis of dermoscopic images may help general practitioners in detecting possible melanoma in its early stages, facilitating diagnosis and treatment.

The segmentation step is very important as it provides the means to isolate the lesion area for better analysis. Image segmentation combines techniques from many fields of image processing such as pattern recognition, scene and image analysis. Segmentation consists of five main approaches namely threshold, edge detection, region-based processing, pixel intensity, and morphological methods ${ }^{3}$. Although many segmentation methods use a grey level image for processing, our proposed method used colour images in order to isolate the three colour layers (i.e., R, G, and $\mathrm{B})$.

The nature of melanoma detection requires the knowledge of the lesion colour as one of the detection criteria. Our method proposed steps to select the best threshold value with morphological operation. The paper is organized as follows: a review of the related work followed by the description of our proposed segmentation algorithm, the experimental results, discussion and comparisons as well as conclusions and future work.

Several methods can be used for image segmentation such as clustering methods ${ }^{4,5}$, threshold method $^{6}$, classifier, region growing ${ }^{7}$, deformable model and Markov random model ${ }^{8,9}$. Segmentation techniques are especially useful in the analysis of medical images. The traditional method for thresholding is Otsu's method, which removes the region with low variation for finding a suitable threshold value to minimize intra-class variance ${ }^{6}$. For every suggested threshold value, the method evaluates the feasibility of this value if used as the threshold. Garnavi et $\mathrm{al}^{1}$ proposed an automatic segmentation procedure using clustering histogram thresholding and then used Otsu's method to separate lesion and healthy skin region from images. The rate of accuracy is 


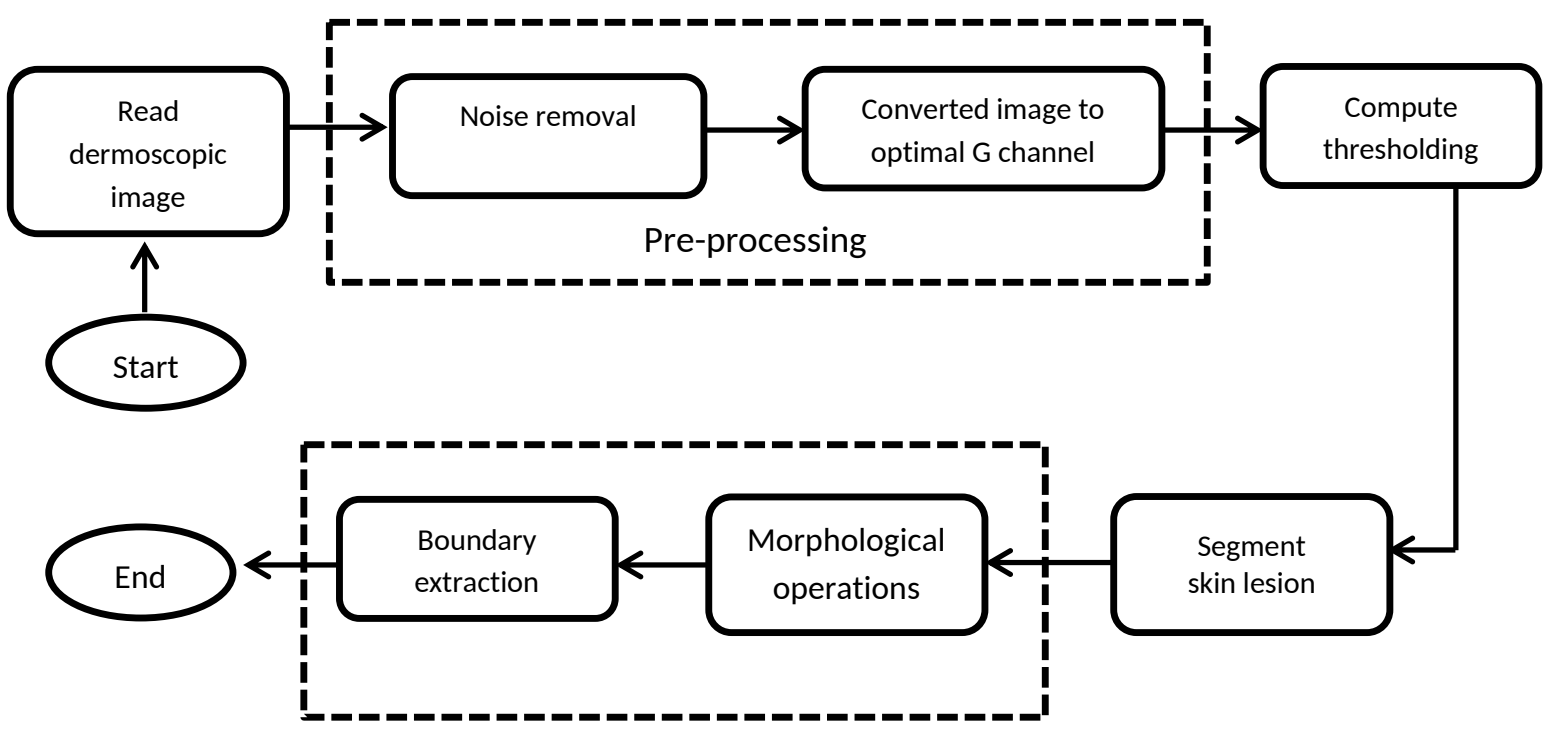

Fig. 1 Flowchart illustrating the steps of the proposed method.

between 92 and 96\%. Ajala Funmilola et al ${ }^{10}$ combined approaches for fuzzy C-mean clustering (FCM) and implemented it on MRI images of human brain. Also, they obtained good results for segmentation. In our previous paper ${ }^{3}$, we combined watershed with optimal wavelet in order to segment the lesion. Most of image segmentation methods are performed on grey level images because the required processing time in grey images is lower than colour images. RGB colour space is useful for displaying but it is not always suitable for scene segmentation and analysis due to the high correlation among the three colour channels $(\mathrm{R}, \mathrm{G}, \mathrm{B})^{11}$. Sadhana and Sanjay ${ }^{12}$ proposed an efficient method to segment melanoma using two methods of clustering, which were K-means and fuzzy C-means. They implemented these methods after they converted RGB image to the YIQ colour space. The luminance component $(\mathrm{Y})$ represents grey-scale information, and the other two components carry the colour information. The YIQ components can be obtained by converting the RGB components of an original image. Razmjooy et $\mathrm{al}^{13}$ proposed a method to convert the colour images with RGB colour space to mode intensity images because the images in their database had varied colour tones and were captured using different devices. In addition, they implemented Kapur's entropy method ${ }^{13}$ to select a good value of thresholding. The skin lesion was then segmented from the healthy skin after implementing morphological operations. However, their segmentation results showed that they were able to obtain good segmentation only for dark and thick regions of the lesion and have left the lighter colour portions of the lesion undetected. Schaefer et $\mathrm{al}^{14}$ solved the problem of low contrast and lack of colour in dermoscopic images because of the different devices. They obtained good results by improving the normalization of colour images in the pre-processing stage. Wang et al ${ }^{15}$ modified watershed and post-processing procedures to segment lesions in dermoscopic images. They obtained an average percentage error of $16 \%$. Moreover, they used edge object value thresholding to remove large light blobs around the lesion boundary.

\section{THE PROPOSED METHOD FOR SEGMENTATION}

In this section, we discussed the processes involved in the proposed method. These processes can be grouped into three major stages (Fig. 1): pre-processing, image segmentation, and post-processing.

\section{Pre-Processing}

The features such as edge, shape, texture, and colour of the lesion can be extracted after completing the segmentation of the image. Prior to the segmentation stage, images have much noise such as hair, variations in brightness, and sometimes small air bubbles. Preprocessing steps were used to eliminate these noise and prepare the images for segmentation. We applied median filter to enhance images with skin lesions. The algorithm of this filter replaces each pixel with the median value of the neighbouring pixels. This method is also useful for removing light reflection in images, as well as other small dotted noise in the background outside the skin area ${ }^{13,16}$. Some researchers ${ }^{17-19}$ have 

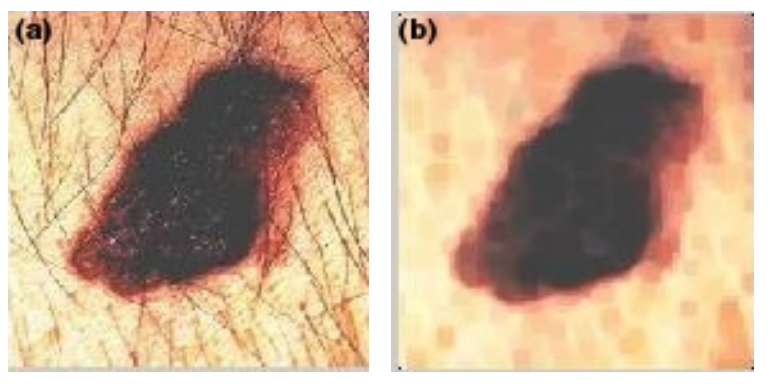

Fig. 2 The removal of thick hair noise from dermoscopic image. (a) Original image, (b) after hair removal.
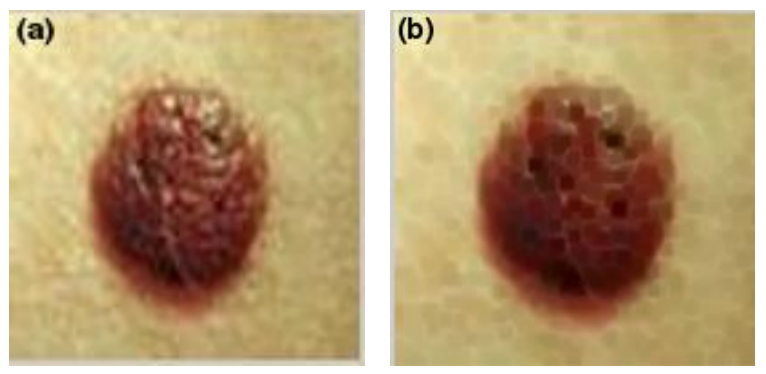

Fig. 3 The light removal from dermoscopic image. (a) Original image, (b) after light removal.

applied the Dullrazor software in order to remove hair. The process of this software consists of three steps: (1) identifying the locations of dark hair, (2) replacing the hair pixels using bilinear interpolation, and (3) smoothing the image in the final result using adaptive median filter. Smaller structures and artefacts can be removed using median filter with different window sizes ${ }^{20,21}$. This method produces reasonable results when removing thin hair from images, but when attempting to remove thick hair or a regions with large amount of hair, the software was found to have also some parts removed of the lesion from the image. In this paper, we implemented morphological operations (closing and eroding) to remove thick hair and reflecting light from devices. Our method was able to remove the hair while leaving the lesion region intact. Next, we applied median filter to reduce the effect of small structures, like thin hair, or scale lines as shown in Fig. 2 and Fig. 3, respectively.

To improve the segmentation of skin lesion, we converted the colour image from RGB colour space with two coordinates $M_{1}(x, y)$ to obtain a new scaled intensity after implementing:

$$
M_{2}(x, y)=\omega M_{1}(x, y),
$$

where $\omega$ is a constant value. $M_{2}(x, y)$ is the image with new scale intensity. Different values of $\omega$ (such

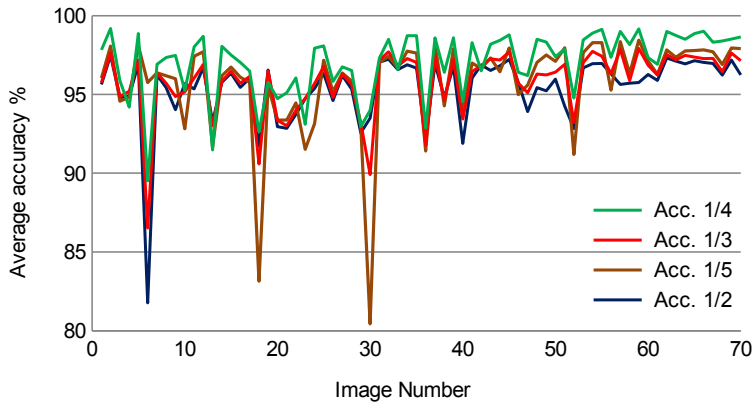

Fig. 4 Comparison of average percentage of accuracy for different values of $\omega$, and $\omega=1 / 4$ showed the best result.

Table 1 The comparison results of the proposed method with different colour channels.

\begin{tabular}{lccc}
\hline Colour channel & Accuracy & Sensitivity & Specificity \\
\hline G & 97.03 & 96.69 & 97.26 \\
B & 95.55 & 94.67 & 96.11 \\
R & 95.87 & 91.17 & 98.19 \\
RG & 92.32 & 78.07 & 98.67 \\
RB & 92.90 & 81.07 & 98.18 \\
GB & 94.38 & 89.66 & 96.39 \\
RGB & 96.48 & 91.40 & 98.37 \\
\hline
\end{tabular}

as 1/2,1/3, 1/4, and 1/5) were applied, shown in Fig. 4, the highest average percentage of accuracy was found at the constant value of $1 / 4$.

Various colour channel combinations were implemented individually ( $R, G, B, R G, R B, G B$, and RGB), and the average percentage of accuracy, sensitivity, and specificity were computed for each of these combinations. The highest average accuracy is found to be the green channel (G-Opt) of 97\% (Table 1). It can be observed that the value for the average percentage of accuracy is between the values of average sensitivity and specificity. This is because the sensitivity indicates the method ability to include all possible pixels that may belong to the lesion, on the other hand, value of average specificity is determined by the method capability to exclude pixels that may not belong to the lesion. The green channel has been chosen for this paper, as it has produced the highest average percentage of accuracy. The histogram of a grey level image is bimodal if it has two peaks; one for the background and the other for the object. Ideally, the object can be isolated from the background easily if the image is clear and free from noise, the threshold value can be found easily at the bottom of the histogram valley ${ }^{22}$. However, in most cases, the histogram for camera captured images can be noisy and have a few peaks, which renders the task 

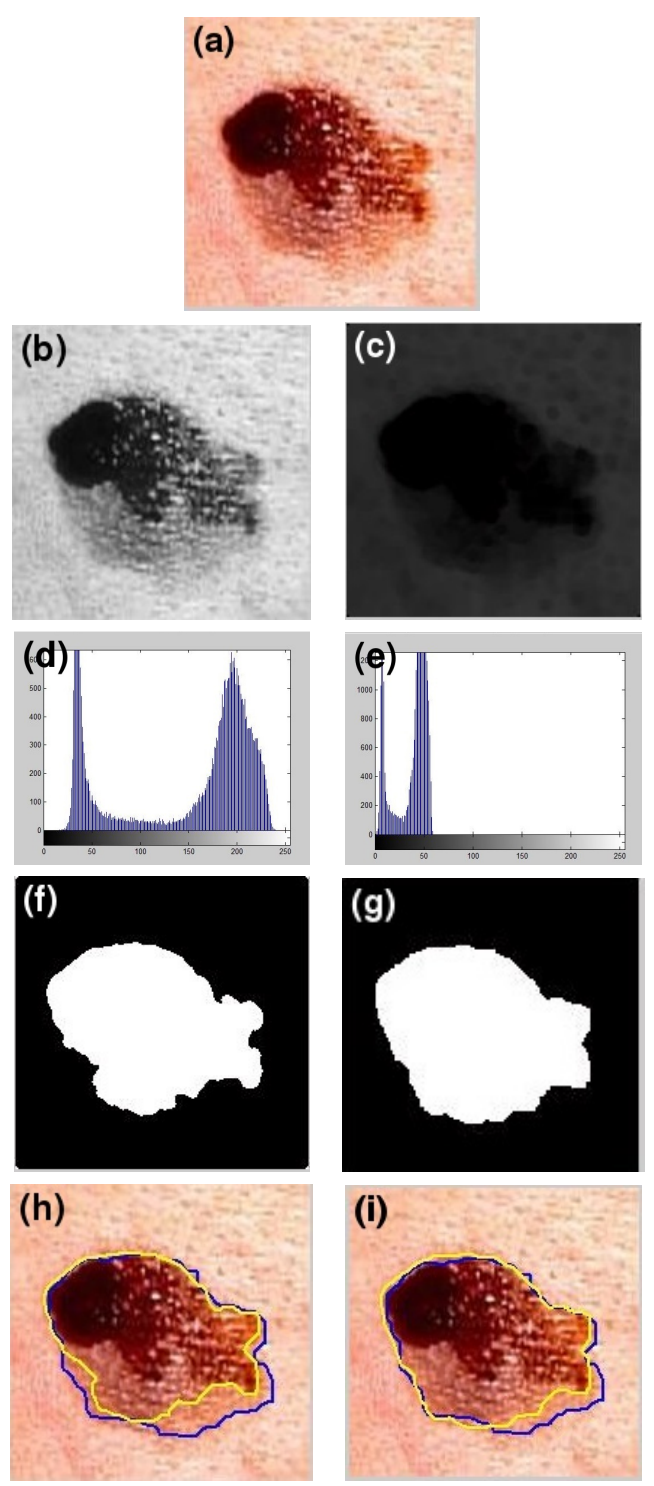

Fig. 5 Histogram (d, e) for both using RGB and the green channel, the black and white labels created after thresholding, and the automatic border (yellow) and manual border (blue) on the original image after segmentation. (a) Original image, (b, d, f, h) RGB (grey level), (c, e, g, i) green channel (G-opt), (f, g) label (BW), and (h, i) auto and manual borders.

of finding the threshold value difficult. When the RGB and the green channel were rescaled to intensity values, the histogram for RGB intensity was found to have a much wider valley compared to the valley produced for the green channel (G-Opt) Fig. 5. By having a clearly defined valley in the histogram, it is possible to determine the correct threshold value for segmentation. The segmentation border results are displayed on the right-hand side of Fig. 5. The yellow line indicates the automatic generated border, and the blue line indicates the professionally determined manual border. The automatic border (yellow lines) created by the green channel intensity in comparison to the RGB intensity has a much closer resemblance to the manually drawn border (blue lines). The result of this comparison was further quantified by the average percentage results shown in Table 1.

\section{Image Segmentation}

The mathematical morphology operation is one of the segmentation approaches used in this paper. The purpose of this technique is to enhance the edges on the lesion boundary, while suppressing the gradients inside the lesion and in the background. The segmentation step is defined as the process of merging pixels with similar features into the same group such as grey level, colour, texture, brightness, and contrast. The threshold value is very important for the medical image segmentation techniques, as it will separate pixels into two classes depending on grey level of medical image. Some of the pixels in one class may have values greater than the threshold, while some pixels in the second class may have intensity values less than the threshold value. The skin lesion region can then be isolated from the background of the dermoscopic image. The white colour is considered skin lesion and the black colour is healthy skin. To create black and white labels we used

$$
M_{3}(x, y)= \begin{cases}1, & \text { if } M_{2}(x, y) \leqslant(\mu-a), \\ 0, & \text { otherwise },\end{cases}
$$

where

$$
\mu=\frac{1}{M N} \sum_{x=1}^{N} \sum_{y=1}^{M} M_{2}(x, y),
$$

and $a$ is a constant with value of $1,3,5$, or 7 . Fig. 6 shows results using various values of $a$. When $a=3$ the average accuracy is at its highest. $M_{2}(x, y)$ is the new scaled intensity for the G-opt channel and $M_{3}(x, y)$ is the black and white binary image.

\section{Post-Processing}

Morphological operations such as closing, eroding, bridging, and filling were used. Smooth images can be obtained by eliminating small holes after the segmentation and by filling in gaps between the smaller areas of the lesion with larger areas and forming a unified lesion boundary. These operations were applied to the images to obtain smooth images, and reduced image contrast by narrowing the histogram peak. As observed in Fig. 7, the results from our method showed 

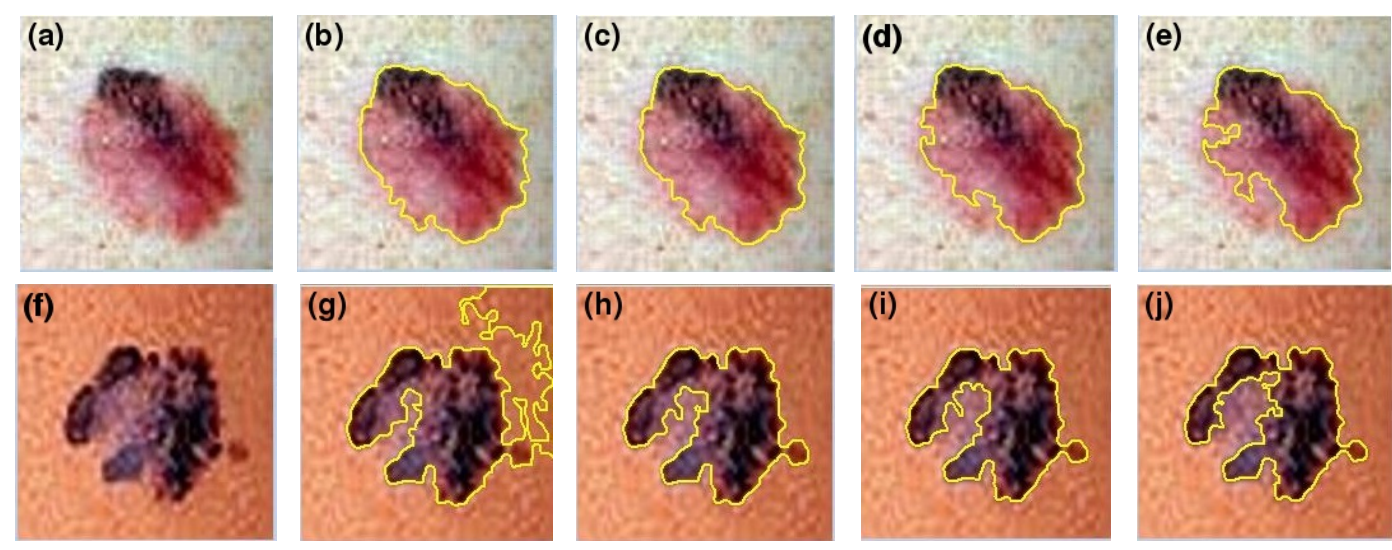

Fig. 6 The segmentation result based on different thresholding values of $a$, highest accuracy results can be obtained when $a=3$; (a-e) image A, (f-j) image B; (a, f) original images, (b, g) $a=1,(\mathrm{c}, \mathrm{h}) a=3,(\mathrm{~d}, \mathrm{i}) a=5$, and $(\mathrm{e}, \mathrm{j}) a=7$.

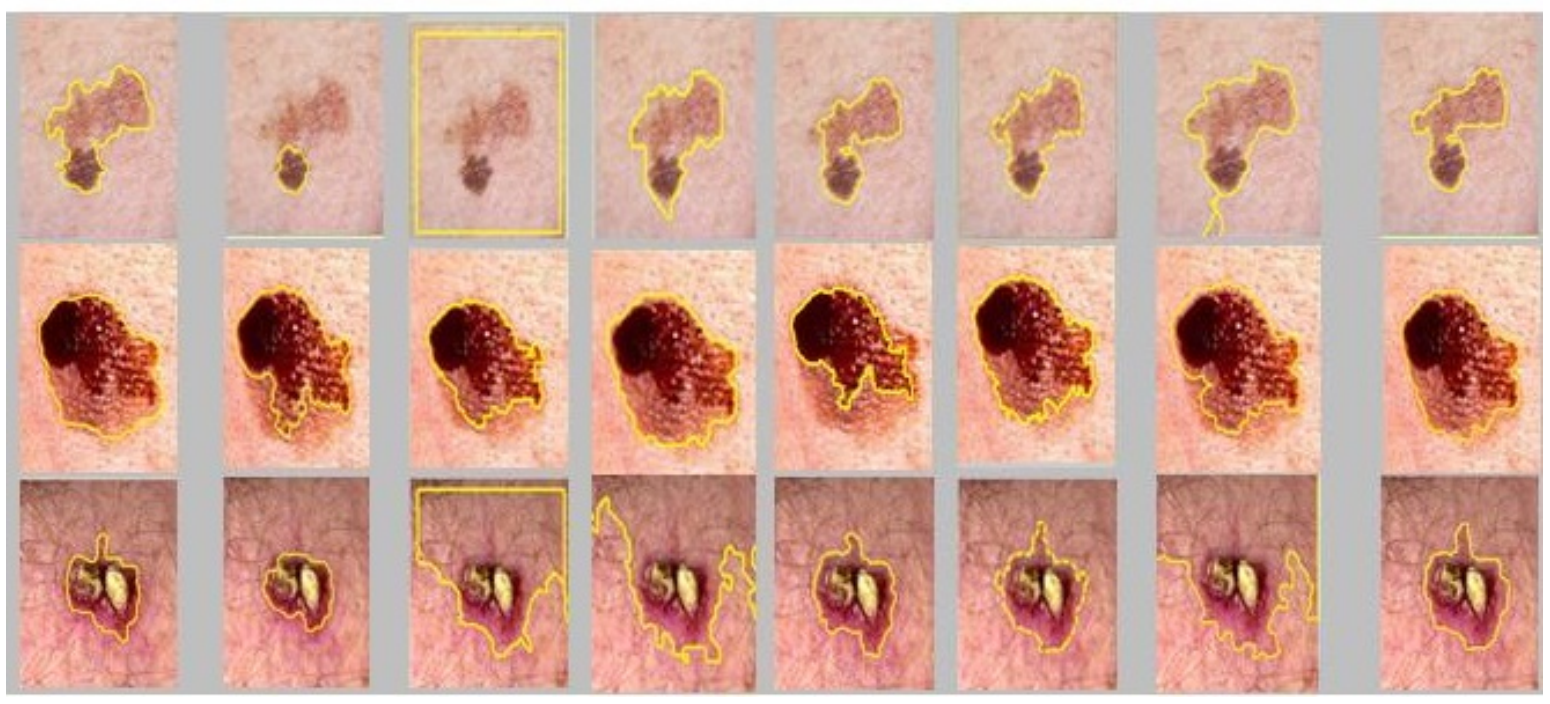

Fig. 7 Comparison of results between manual border images and the segmentation images from proposed method with other researchers' methods. Image A (top), image B (middle), and image C (bottom). Columns from left to right: original image with manual borders, Razmjooy et al, Wang et al, Gerald et al, FCM, Otsu method, combined watershed and wavelet (bior 3.3), and proposed method G-opt.

that it was not only capable of segmenting the dark regions of the lesion, but was also able to detect the lighter part of the skin lesions. This enhanced detection capability led to an improved average percentage of segmentation accuracy compared to other methods (Table 2).

\section{EXPERIMENTAL RESULTS AND EVALUATION}

We collected 70 dermoscopic images of both malignant and benign skin lesions with size $200 \times 200$ pixels. As the images were obtained from various sources, they contain different lighting conditions, colour, and tone. This is to test our method on a wider range of dermoscopic images. Before we applied our method, we converted the images into grey level, and rescaled them into new intensities. In this case, we tested (1) with different values for $\omega$ and the best value was found to be 1/4 (Fig. 4). As for segmentation, different values of $a$ were tested. When the value of $a$ was above 3 , only the dark area of the lesion could be detected; conversely, when the value of $a$ was below 3 , some healthy skin regions were detected as skin lesions. The best value for $a$ was found to be 3 as it gave the highest average of accuracy (Fig. 6).

In the experiment, the ground truth were borders manually drawn by dermatologists. These were compared with the automatic borders produced by 
Table 2 The comparison results of the proposed method with different colour channels.

\begin{tabular}{lrrr}
\hline Methods & Accuracy & Sensitivity & Specificity \\
\hline Razmjooy et al & 91.49 & 82.90 & 95.38 \\
Wang et al & 95.14 & 76.87 & 98.40 \\
Gerald et al & 94.77 & 89.44 & 97.79 \\
FCM & 92.12 & 75.92 & 99.52 \\
Otsu & 95.33 & 97.15 & 93.81 \\
Watershed and wavelet $^{*}$ & 94.61 & 88.60 & 98.21 \\
Proposed method & 97.03 & 96.69 & 97.26 \\
\hline
\end{tabular}

* Watershed and wavelet (bior 3.3).

the proposed method. Three statistical metrics were used to evaluate our proposed method by comparing automatic borders with manual borders. They were sensitivity, specificity, and accuracy. These metrics are calculated from the following expressions:

$$
\begin{aligned}
\text { Sensitivity } & =\frac{\mathrm{TP}}{\mathrm{TP}+\mathrm{FN}}, \\
\text { Specificity } & =\frac{\mathrm{TN}}{\mathrm{TN}+\mathrm{FP}}, \\
\text { Accuracy } & =\frac{\mathrm{TN}+\mathrm{TP}}{\mathrm{TN}+\mathrm{TP}+\mathrm{FP}+\mathrm{FN}},
\end{aligned}
$$

where $\mathrm{TP}$ is the number of overlapping pixels in the lesion, $\mathrm{TN}$ is the number of overlapping pixels outside the lesion, FP is the number of overlapping pixels between the automatically labelled lesion and those outside the manually labelled lesion, and FN is the number of overlapping pixels between the manually labelled lesion and those outside the automatically labelled lesion ${ }^{3}$.

\section{DISCUSSION AND COMPARISON}

We have compared our results to that using other methods and techniques (Fig. 7). Three lesion images which are representative of some of the different variations of lesions were chosen for comparison. Image A in Fig. 7 presented a lesion where the skin tone is very close to the healthy skin, the result is a lowered contrast between lesion and healthy skin area. Whereas, image B shows a relatively typical lesion that has a colour tone much different from that of the healthy skin. As for image $\mathrm{C}$, it shows a lesion with lighter colour than the surrounding healthy skin. Among the techniques compared in Fig. 7, Razmjooy et $\mathrm{al}^{13}$, Wang et $\mathrm{al}^{15}$, and Gerald et al ${ }^{14}$ were methods designed specifically for lesion segmentation, $\mathrm{FCM}^{4}$ and the Otsu methods ${ }^{6}$ were established as general purpose segmentation techniques that have been widely used in other areas of the application. As for the two rightmost methods on Fig. 7, the combined watershed and wavelet method ${ }^{3}$ was our previously proposed method. The G-Opt method is the proposed method for this paper. Although most of the methods here were capable of isolating the majority of lesion from the healthy skin, many of the techniques, including our previous combined watershed and wavelet method, left some parts of the lighter coloured lesion regions undetected. Whereas, in some cases for images $\mathrm{A}$ and $\mathrm{C}$, large regions of the healthy skin were segmented as lesions. Although some techniques compared here have shown high rates of specificity as seen in Table 2, however, the results would not segment the lighter coloured regions of the lesion. Conversely, some of these techniques that have provided high sensitivity were found to have misidentified regions of healthy skin as a part of the lesion. The proposed method was able to delineate the lesion skin area in images A and B of Fig. 7. In image $\mathrm{C}$, there are large amounts of noise from the existence of hair around the lesion. Our preprocessing steps allowed the removal of noise from the images before progressing forward with segmentation, this also provided a more accurate result.

\section{CONCLUSION AND FUTURE WORK}

This paper presented an improved algorithm for skin lesion segmentation. This algorithm consisted of preprocessing to remove the thin hair and small noise from skin images. Before segmenting the skin lesion, the original RGB images were rescaled to new intensities, to be able to solve the problem of low contrast and variation of colour in the dermoscopic images. We compared our proposed method with other methods and satisfactory results were obtained. From the results, the proposed method achieved the highest average accuracy of $97 \%$ for the 70 dermoscopic images with both malignant and benign melanoma from multiple sources. In the future, we plan to further enhance and extend the proposed technique to other types of skin lesions.

Acknowledgements: The authors thank Dr Joaquim M. da Cunha Viana and Mr Navid Razmjooy for providing the dermoscopic images used in this study. We also thank the dermatology specialist, Dr Mohammed Ahmed, for providing the required information and references for this work. This study has been funded by the University of Malaya, RG104-12ICT.

\section{REFERENCES}

1. Garnavi R, Aldeen M, Celebi ME, Varigos G, Finch S (2011) Border detection in dermoscopy images using hybrid thresholding on optimized color channels. Comput Med Imag Graph 35, 105-15. 
2. American Cancer Society (2014) What are the survival rates for melanoma skin cancer by stage? http://www.cancer.org/cancer/skincancer-melanoma/ detailedguide/melanoma-skin-cancer-survival-rates.

3. Abbas AA, Tan WH, Guo XN (2012) Combined optimal wavelet filters with morphological watershed transform for the segmentation of dermoscopic skin lesions. In: Lecture Notes in Computer Science 7458, PRICAI 2012: Trends in Artificial Intelligence, pp 722-7.

4. Sikka K, Sinha N, Singh PK, Mishra AK (2009) A fully automated algorithm under modified FCM framework for improved brain MR image segmentation. Magn Reson Imag 27, 994-1004.

5. Dou W, Ren Y, Chen Y, Ruan S, Bloyet D, Constans JM (2005) Histogram-based generation method of membership function for extracting features of brain tissues on MRI images. In: Lecture Notes in Computer Science 3613, Fuzzy Systems and Knowledge Discovery, pp 189-94.

6. Otsu N (1979) A threshold selection method from graylevel histograms. IEEE Trans Syst Man Cybern 9, 62-6.

7. Dhawan AP, Sim A (1992) Segmentation of images of skin lesions using color and texture information of surface pigmentation. Comput Med Imag Graph 16, 163-77.

8. Ghassan H (2001) Towards intelligent deformable models for medical image analysis. $\mathrm{PhD}$ thesis, Chalmers Univ of Technology Gøteborg, Sweden.

9. Bauer S, Nolte LP, Reyes M (2011) Segmentation of brain tumour images based on atlas-registration combined with Markov-random-field lesion growth model. In: IEEE International Symposium on Biomedical Imaging: From Nano to Macro, Chicago, pp 2018-21.

10. Ajala Funmilola A, Oke OA, Adedeji TO, Alade OM, Adewusi EA (2012) Fuzzy k-c-means clustering algorithm for medical image segmentation. J Inform Eng Appl 2, 21-32.

11. Pietikainen $\mathrm{M}$, Nieminen $\mathrm{S}$, Marszalec E, Ojala $\mathrm{T}$ (1996) Accurate colour discrimination with classification based on feature distributions. In: Proceedings of the 13th International Conference on Pattern Recognition, vol. 3, pp 833-8.

12. Sadhana PB, Sanjay P (2011) Segmentation of melanoma skin cancer images based on clustering techniques. Res J Comput Syst Eng 2, 287-95.

13. Razmjooy N, Mousavi BS, Soleymani F, Khotbesara M (2012) A computer-aided diagnosis system for malignant melanomas. Neural Comput Appl 23, 2059-71.

14. Schaefer G, Rajab MI, Celebi ME, Iyatomi H (2011) Colour and contrast enhancement for improved skin lesion segmentation. Comput Med Imag Graph 35, 99-104.

15. Wang H, Moss RH, Chen X, Stanley RJ, Stoecker WV, Celebi ME, Malters JM, Grichnik JM, Marghoob AA, Rabinovitz HS, Menzies SW, Szalapski TM (2011) Modified watershed technique and post-preprocessing and post-processing for segmentation of skin lesions in dermoscopy images. Comput Med Imag Graph 35, 116-20.

16. Korotkov K, Garcia R (2012) Computerized analysis of pigmented skin lesions: A review. Artif Intell Med 56, 69-90.

17. Lee $\mathrm{T}, \mathrm{Ng} \mathrm{V}$, Gallagher R, Coldman A, McLean D (1997) DullRazor: A software approach to hair removal from images. Comput Biol Med 27, 533-43.

18. Zhou H, Chen M, Gass R, Rehg JM, Ferris L, Ho J, Drogowski L (2008) Feature-preserving artifact removal from dermoscopy images. In: Proceedings of SPIE 6914, Medical Imaging 2008: Image Processing, 69141B.

19. Nguyen NH, Lee TK, Atkins MS (2010) Segmentation light and dark hair in dermoscopic images: a hybrid approach using a universal kernel. In: Proceedings of SPIE 7623, Medical Imaging 2010: Image Processing, $76234 \mathrm{~N}$

20. Zagrouba E, Barhoumi W (2004) A preliminary approach for the automated recognition of malignant melanoma. Image Anal Stereol 23, 121-35.

21. Chiem A, Al-Jumaily A, Khushaba RN (2007) A novel hybrid system for skin lesion detection. In: Proceedings of the 3rd International Conference on Intelligent Sensors, Sensor Networks and Information, ISSNIP 2007, pp 567-72.

22. Kapur JN, Sahoo PK, Wong AKC (1985) A new method for gray-level picture thresholding using the entropy of the histogram. Comput Vis Graph Image Process 29, 273-85. 